\title{
3.2 Thermal regime control of thermoelectric cooling devices when minimizing a complex of four basic parameters
}

Thermoelectric systems for ensuring the thermal conditions of heat-loaded electronic components are the main ones in conditions of the need to meet stringent requirements for weight, dimensions, reliability, and dynamic characteristics. The control of the thermal regime of a thermoelectric cooling device (TEC) in several cases is determined by a set of four main parameters $\left(n I \lambda / \lambda_{0} \tau\right)$. The choice of the TEC design is the choice of the geometry of the thermoelement legs (ratio $l / S$ ), their number $n$, the value of the operating current $I$ in conjunction with the reliability indicators $\lambda / \lambda_{0}$ and the dynamics of functioning $\tau$. With a rational design of the TEC, one should strive to reduce the number of thermoelements $n$ the value of the operating current $I$, the relative failure rate $\lambda / \lambda_{0}$ and the time to enter the stationary mode of operation $\tau$.

These basic parameters are interconnected, despite their inconsistency. So, with a constant heat load $Q_{0}$ and a fixed geometry of thermoelement legs (ratio $l / S$ ):

- a decrease in the number of thermoelements $n$ can be compensated by an increase in the relative operating current $B$. At the same time, the failure rate $\lambda / \lambda_{0}$ changes and the time to reach the stationary operating mode decreases $\tau$;

- an increase in the number of thermoelements $n$ can be compensated for by

a decrease in the value of the relative operating current $B$. In this case, the intensity of failures $\lambda / \lambda_{0}$ changes and the time $\tau$ to reach the stationary mode of operation increases.

The relative rate $\lambda / \lambda_{0}$ depends on the numbers of thermocouples $n$ and the relative operating current $B$ at s fixed temperature drop $\Delta T$.

The time to reach the stationary mode $\tau$ of operation depends on the value of the relative operating current $B$, mass and heat capacity of structural and technological elements (CHP) on the heat-absorbing junction of the TPU at a fixed temperature drop 
$\Delta T$. Thus, there is an optimal value of the relative operating current $B_{\text {opt }}$ corresponding to the minimum value of the complex $\left(n I \lambda / \lambda_{0} \tau\right)_{\min }$, which is the subject of this work.

The number of thermoelements $n$ of a single-stage TEC can be determined from the ratio:

$$
n=\frac{Q_{0}}{I_{\max }^{2} R_{\mathrm{K}}\left(2 B_{\mathrm{K}}-B_{\mathrm{K}}^{2}-\Theta\right)},
$$

where $Q_{0}-$ heat load value, W;

$I_{\operatorname{maxK}}=\frac{\bar{e}_{\mathrm{K}} T_{0}}{R_{\mathrm{K}}}-$ maximum operating current, $\mathrm{A}$.

$\bar{e}_{\mathrm{K}}-$ average value of thermoEFM coefficient of thermoelement leg at the end of the cooling process, $\mathrm{V} / \mathrm{K}$;

$R_{\mathrm{K}}=\frac{l}{\bar{\sigma} S}-$ electrical resistance of the thermoelement branch, Ohm;

$l$ and $S-$ respectively, the height $l$ and the cross-sectional area $S$ of the thermoelement leg;

$\bar{\sigma}-$ average value of thermoelement branch electrical conductivity, S/cm;

$T_{0}-$ heat-absorbing junction temperature, $\mathrm{K}$;

$B_{\mathrm{K}}=\frac{I}{I_{\operatorname{maxK}}}-$ relative operating current at the end of the cooling process;

$\Theta=\frac{T-T_{0}}{\Delta T_{\max }}-$ relative temperature difference;

$T$ - heat-generating junction temperature, $\mathrm{K}$;

$\Delta T_{\max }=0,5 \bar{z} T_{0}^{2}-$ maximum temperature drop, $\mathrm{K} ;$

$\bar{z}$ - average value of the efficiency of the initial thermoelectric materials in the module, 1 / K.

The power consumption $W_{\mathrm{K}}$ of the TEC can be determined from the expression:

$$
W_{\mathrm{K}}=2 n \cdot I_{\operatorname{maxK}}^{2} \cdot R_{\mathrm{K}} \cdot B_{\mathrm{K}}\left(B_{\mathrm{K}}+\frac{\Delta T_{\max }}{T_{0}} \Theta\right) .
$$


Voltage drop $U_{\mathrm{K}}$

$U_{\mathrm{K}}=\frac{W_{\mathrm{K}}}{I}$.

The coefficient of performance $E$ can be calculated from the expression:

$E=\frac{Q_{0}}{W_{\mathrm{K}}}$

The relative magnitude of the failure rate $\lambda / \lambda_{0}$ can be determined from the expression [200]:

$$
\lambda / \lambda_{0}=n B_{\mathrm{K}}^{2}(\Theta+C) \frac{\left(B_{\mathrm{K}}+\frac{\Delta T_{\max }}{T_{0}} \Theta\right)^{2}}{\left(1+\frac{\Delta T_{\max }}{T_{0}} \Theta\right)^{2}} \cdot K_{\mathrm{T}},
$$

where $C=\frac{Q_{0}}{n I_{\max }^{2} R_{\mathrm{K}}}-$ relative heat load,

$K_{\mathrm{T}}-$ significant coefficient of reduced temperatures [200].

The probability of failure-free operation $P$ of the TEC can be determined from the expression:

$$
P=\exp [-\lambda t]
$$

where $t$ - assigned resource, h.

The ratio for determining the time of reaching the stationary mode of operation $\tau$ can be represented as [201]:

$$
\tau=\frac{m_{0} C_{0}+n \sum_{i} m_{i} C_{i}}{n K_{L}\left(1+2 B_{\mathrm{K}} \frac{\Delta T_{\max }}{T_{0}}\right)} \ln \frac{\gamma B_{\mathrm{H}}\left(2-B_{\mathrm{H}}\right)}{2 B_{\mathrm{K}}-B_{\mathrm{K}}^{2}-\Theta},
$$

where $\gamma=\frac{I_{\max }^{2} R_{\mathrm{H}}}{I_{\max }^{2} R_{\mathrm{K}}}$

$m_{0} C_{0}$ - mass and heat capacity of the object to be cooled. In our case $m_{0} C_{0} \rightarrow 0$ (there is no cooling object). 
$\sum_{i} m_{i} C_{i}-$ the total value of the product of the heat capacity and the mass of the constituent structural and technological elements (CHP) on the heat-absorbing junction of the module at a given $l / S$;

$R_{\mathrm{H}}$ - electrical resistance of the thermoelement leg at the beginning of the cooling process, Ohm;

$B_{\mathrm{H}}=\frac{I}{I_{\operatorname{maxH}}}-$ relative operating current at the beginning of the cooling process, $\tau=0$

$I_{\operatorname{maxH}}=\frac{\bar{e}_{\mathrm{H}} \cdot T}{R_{\mathrm{H}}}-$ maximum operating current at the beginning of the cooling process, A.

Provided that the currents are equal at the beginning and at the end of the cooling process:

$I=B_{\mathrm{K}} I_{\operatorname{maxK}}=B_{\mathrm{H}} I_{\operatorname{maxH}}$.

Then the expression for the complex $\left(n I \lambda / \lambda_{0} \tau\right)$ can be written in the form:

$$
K=n I \lambda / \lambda_{0} \tau=\frac{Q_{0}^{2} B_{\mathrm{K}}^{3}(\Theta+C)\left(B_{\mathrm{K}}+\frac{\Delta T_{\max }}{T_{0}} \Theta\right)^{2} K_{\mathrm{T}} \sum_{i} m_{i} C_{i} \ln \frac{\gamma B_{\mathrm{H}}\left(2-B_{\mathrm{H}}\right)}{2 B_{\mathrm{K}}-B_{\mathrm{K}}^{2}-\Theta}}{I_{\text {max }_{\mathrm{K}}}^{3} R_{\mathrm{K}}^{2}\left(2 B-B^{2}-\Theta\right)^{2}\left(1+\frac{\Delta T_{\max }}{T_{0}} \Theta\right)^{2} K_{L}\left(1+2 B_{\mathrm{K}} \frac{\Delta T_{\max }}{T_{0}}\right)},
$$

where $K_{L}=\frac{\kappa \cdot S}{l}, \kappa$-average value of the thermal conductivity coefficient, $\frac{\mathrm{w}}{\mathrm{cm} \cdot \mathrm{K}}$, or in the form convenient for differentiation:

$A=\frac{K I_{\text {max }_{\mathrm{K}}}^{3} R_{\mathrm{K}}^{2}\left(1+\frac{\Delta T_{\max }}{T_{0}} \Theta\right)^{2} K_{L}}{Q_{0}^{2}(\Theta+C) K_{\mathrm{T}} \sum_{i} m_{i} C_{i}}=\frac{B^{3}\left(B_{\mathrm{K}}+\frac{\Delta T_{\max }}{T_{0}} \Theta\right)^{2} \cdot \ln \frac{\gamma B_{\mathrm{H}}\left(2-B_{\mathrm{H}}\right)}{2 B_{\mathrm{K}}-B_{\mathrm{K}}^{2}-\Theta}}{\left(2 B_{\mathrm{K}}-B_{\mathrm{K}}^{2}-\Theta\right)^{2}\left(1+2 B_{\mathrm{K}} \frac{\Delta T_{\max }}{T_{0}}\right)}$ 
From the condition $\frac{d A}{d B}=0$, by means of simple transformations using the methods of successive approximations with an error of no more than $1 \%$, we obtain the ratio for determining the optimal value of the relative operating current $B_{\text {opt } K}$ corresponding to the minimum value of the complex $\left(n I \lambda / \lambda_{0} \tau\right)_{\min }$ :

$$
B_{\text {optK }}=\frac{\Theta}{2+\frac{T_{\max }}{T_{0}}}\left[1+\frac{\Delta T_{\max }}{T_{0}}\left(2+\frac{\Delta T_{\max }}{T_{0}} \Theta\right)\right]
$$

which corresponds to the regime $E_{\max }$.

The results of calculating the functional $A=f\left(B_{\mathrm{K}}\right)$ dependence for various temperature drops $\Theta$, depending on the relative operating current $B_{\mathrm{K}}$, are presented in Fig. 1.

The functional dependence $A=f(B)$ (Fig. 1) has a minimum for various temperature drops $\Theta$ at $T=300 \mathrm{~K} ; Q_{0}=0,5 \mathrm{~W} ; l / S=4,5$. So, for example, when $\Theta=0,5$ the value $A=0,764$ at $B_{o p t}=0,55$. 


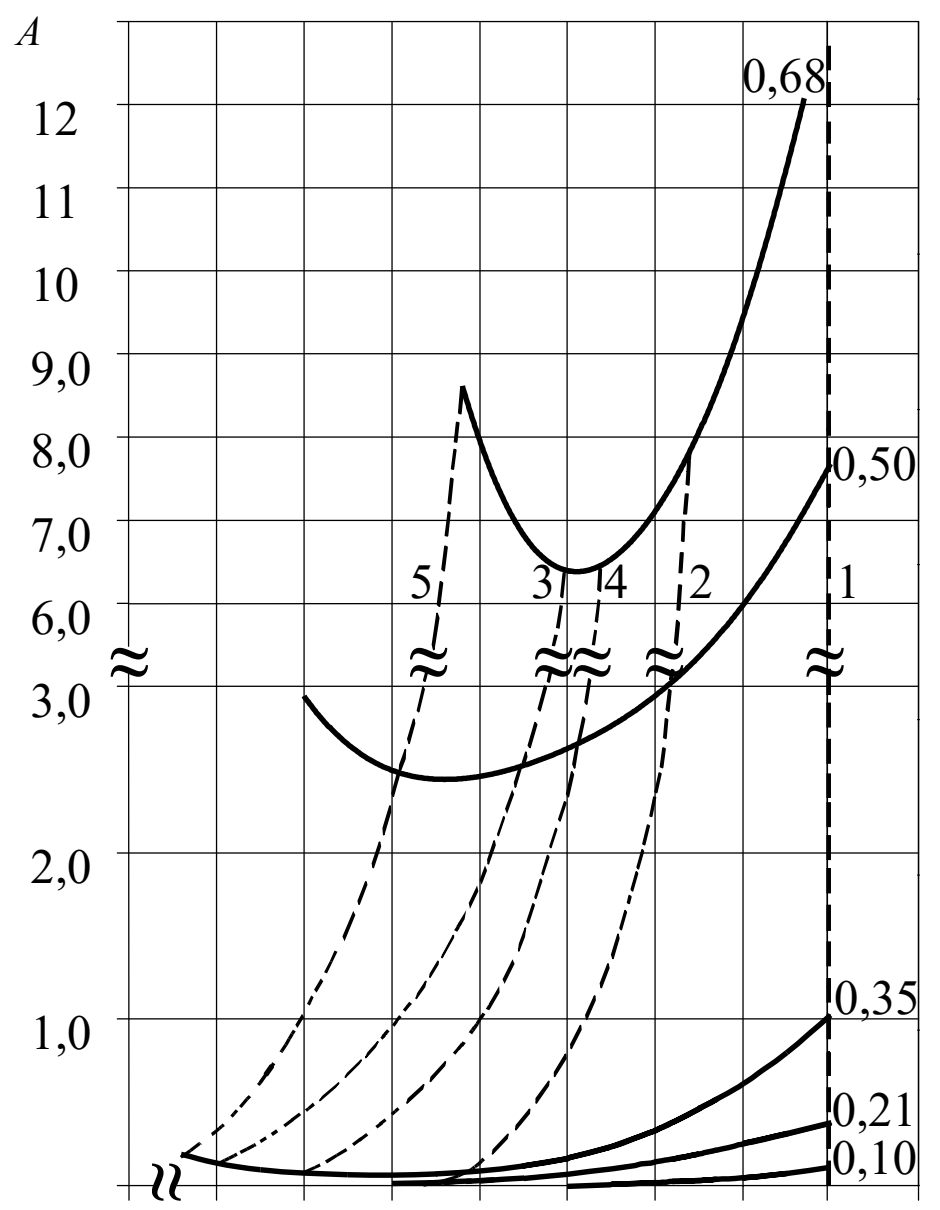

$\begin{array}{llllllllll}0 & 0,3 & 0,4 & 0,5 & 0,6 & 0,7 & 0,8 & 0,9 & 1,0 & B\end{array}$

Fig. 1. Dependence of the value $A$ of a single-stage TEC on the relative operating current $B$ for various relative temperature drops $\Theta$ for $\mathrm{T}=300 \mathrm{~K}, \Theta=0.5 \mathrm{~W}, 1 / \mathrm{S}=4.5$. 1 - mode $Q_{0 \max } ; 2-$ mode $(n I)_{\min } ; 3$ - mode $\left(n I \lambda / \lambda_{0}\right)_{\min } ; 4$ - mode $\left(n I \lambda / \lambda_{0} \tau\right)_{\min } ; 5$ - mode $\lambda_{\min }$.

The dotted line indicated the locus of the point corresponding to different current operating modes: 1 - mode $Q_{0 \max }, 2-\operatorname{mode}(n I)_{\min }, 3$ - mode $\left(n I \lambda / \lambda_{0}\right)_{\min }, 4$ - mode $\left(n I \lambda / \lambda_{0} \tau\right)_{\min }, 5-$ mode $\lambda_{\min }$.

Figure 2 shows the dependence of the optimal relative operating current $B_{\text {opt }}$ for various complexes $(n I)_{\min },\left(n I \lambda / \lambda_{0}\right)_{\min }$, and $\left(n I \lambda / \lambda_{0} \tau\right)_{\min }$ on the relative temperature difference $\Theta$. 


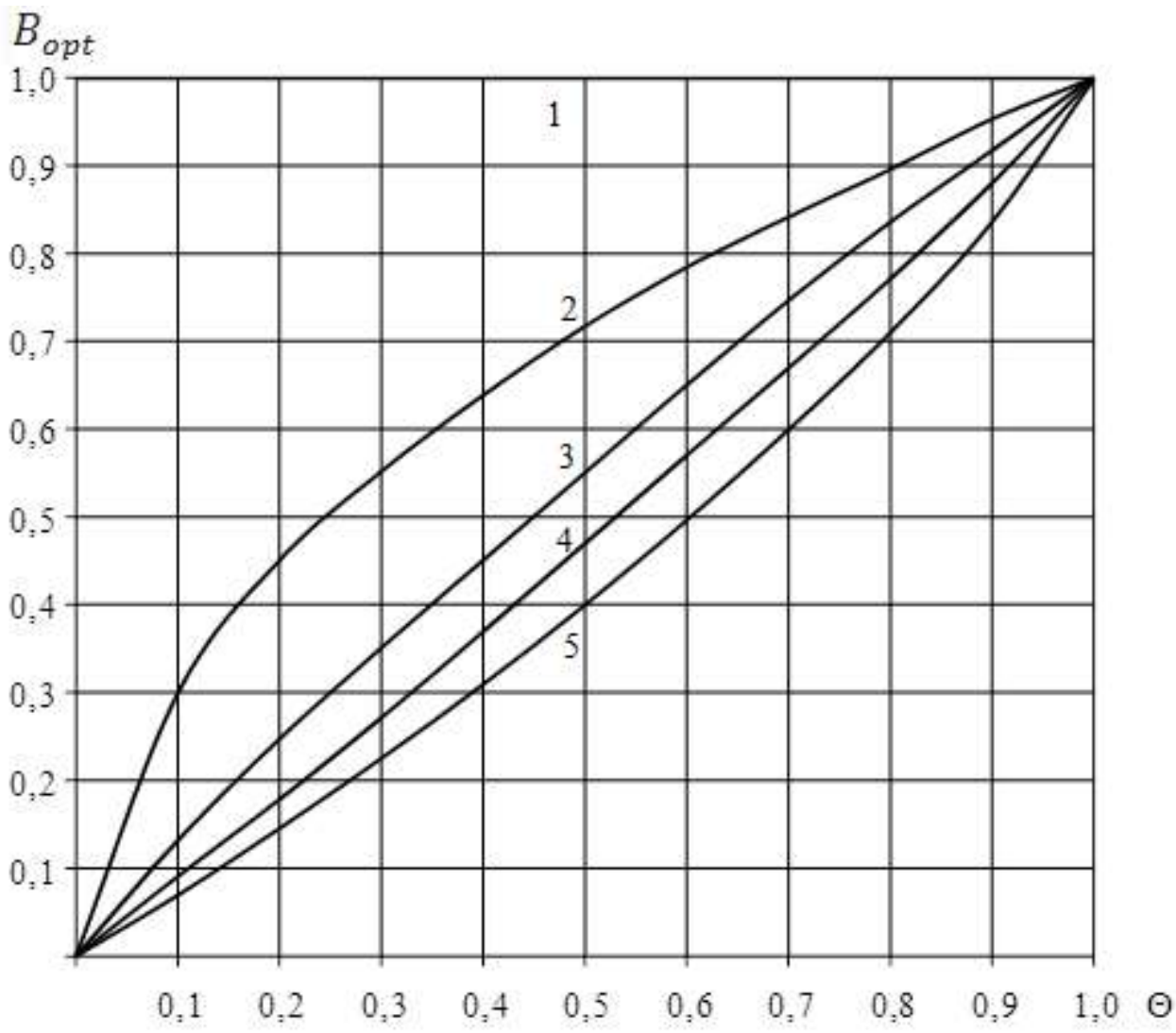

Fig. 2. Dependence of the optimal relative operating current $B_{\text {опт }}$ of a single-stage TEC on the relative temperature drop $\Theta$ for different operating modes at $T=$ $300 \mathrm{~K}, l / s=4,5, Q_{0}=0,5 \mathrm{~W} .1-\operatorname{mode} Q_{0 \max } ; 2-\operatorname{mode}(n I)_{\min } ; 3-$ mode $\left(n I \lambda / \lambda_{0} \tau\right)_{\min } ; 4-$ mode $\left(n I \lambda / \lambda_{0}\right)_{\min } ; 5$ - mode $\lambda_{\min }$.

The results of calculations of the main parameters, reliability indicators and the time to reach the stationary mode of operation of single-stage TEC at $\Delta T=40 \mathrm{~K} ; Q_{0}=0,5 \mathrm{~W}$; and different geometry of thermoelement legs; 10;20;40 and various complexes $l / S=4,5 ; 10 ; 20 ; 40$ are shown in Table 1. 
Table 1

The results of calculations of the main parameters, reliability indicators and the time to reach the stationary mode of operation of single-stage TEC at $\Delta T=40 \mathrm{~K} ; Q_{0}=0,5 \mathrm{~W}$ $l / S=4,5 ; 10 ; 20 ; 40 ; T=300 \mathrm{~K} ; \Delta T_{\max }=79,8 \mathrm{~K} ; \Theta=0,5 ; Q_{0}=0,5 \mathrm{~W}$.

\begin{tabular}{|c|c|c|c|c|c|c|c|c|c|c|c|c|c|c|c|c|c|}
\hline$\lesssim$ & $\begin{array}{c}\text { Operation } \\
\text { mode }\end{array}$ & $B$ & $\begin{array}{l}R \cdot 10 \\
\text { Ohm }\end{array}$ & $\begin{array}{c}I_{\max }, \\
\mathrm{A}\end{array}$ & $\begin{array}{l}n, \\
\text { pc. }\end{array}$ & $\begin{array}{c}W \\
\mathrm{~W}\end{array}$ & $\begin{array}{c}U, \\
\text { B }\end{array}$ & $E$ & $\begin{array}{l}I \\
\mathrm{~A}\end{array}$ & $n I$ & $n I \lambda / \lambda_{0}$ & $\left(n I \lambda / \lambda_{0} \tau\right)_{\min }$ & $\begin{array}{c}\tau \\
\mathrm{s}\end{array}$ & $\begin{array}{l}N \\
\mathrm{~W} \cdot \mathrm{s}\end{array}$ & $\lambda / \lambda_{0}$ & $\begin{array}{c}\lambda \cdot 10^{8} \\
1 / \mathrm{h}\end{array}$ & $P$ \\
\hline \multirow{5}{*}{4,} & $Q_{0 \max }$ & 1,0 & \multirow{5}{*}{4,55} & \multirow{5}{*}{11,1} & 1,8 & 2,3 & 0,21 & 0,22 & 11,1 & 20 & 32,0 & 250 & 7,8 & 17,9 & 1,6 & 4,8 & 0,99953 \\
\hline & $(n I)_{\min }$ & 0,71 & & & 2,3 & 1,46 & 0,19 & 0,34 & 8,0 & 18,4 & 8,1 & 74,5 & 9,2 & 13,4 & 0,44 & 1,53 & 0,99985 \\
\hline & $\left(n I \lambda / \lambda_{0} \tau\right)_{\mathrm{mi}}$ & 0,53 & & & 3,2 & 1,30 & 0,22 & 0,38 & 5,9 & 18,9 & 4,9 & 58,4 & 11,9 & 15,5 & 0,26 & 0,77 & 0,999923 \\
\hline & $\left(n I \lambda / \lambda_{0}\right)_{\min }$ & n 0,47 & & & 4,1 & 1,35 & 0,26 & 0,37 & 5,2 & 21,3 & 4,11 & 57,1 & 13,9 & 18,8 & 0,193 & 0,58 & 0,999943 \\
\hline & $\lambda_{\min }$ & 0,40 & & & 6,6 & 1,62 & 0,34 & 0,31 & 4,8 & 31,7 & 4,9 & 78,0 & 16,0 & 25,9 & 0,154 & 0,462 & 0,999954 \\
\hline \multirow{5}{*}{10} & $Q_{0 \max }$ & 1,0 & \multirow{5}{*}{10,1} & \multirow{5}{*}{5,02} & 3,9 & 2,30 & 0,46 & 0,22 & 5,02 & 19,6 & 78,3 & 50,2 & 6,4 & 14,7 & 4,0 & 12,0 & 0,99880 \\
\hline & $(n I)_{\min }$ & 0,71 & & & 4,7 & 1,46 & 0,41 & 0,34 & 3,6 & 16,9 & 20,8 & 160,0 & 7,7 & 11,2 & 1,23 & 3,7 & 0,99963 \\
\hline & $\left(n I \lambda / \lambda_{0} \tau\right)_{\mathrm{mi}}$ & 0,53 & & & 7,0 & 1,30 & 0,49 & 0,38 & 2,67 & 18,7 & 10,5 & 107,1 & 10,2 & 13,3 & 0,56 & 1,68 & 0,99983 \\
\hline & $\left(n I \lambda / \lambda_{0}\right)_{\min }$ & n 0,47 & & & 9,0 & 1,34 & 0,57 & 0,37 & 2,36 & 21,2 & 8,9 & 107,0 & 12,0 & 16,1 & 0,42 & 1,27 & 0,99987 \\
\hline & $\lambda_{\min }$ & 0,40 & & & 12,0 & 1,62 & 0,81 & 0,31 & 2,0 & 24,0 & 8,6 & 121,0 & 14,0 & 22,7 & 0,36 & 1,1 & 0,99990 \\
\hline \multirow{5}{*}{20} & $Q_{0 \max }$ & 1,0 & \multirow{5}{*}{20,2} & \multirow{5}{*}{2,51} & 7,9 & 2,32 & 0,92 & 0,22 & 2,51 & 19,8 & 123 & 738 & 6,0 & 13,9 & 6,2 & 18,5 & 0,9982 \\
\hline & $(n I)_{\min }$ & 0,71 & & & 10,3 & 1,46 & 0,81 & 0,34 & 1,8 & 18,5 & 37,1 & 274,4 & 7,4 & 10,8 & 2,0 & 6,0 & 0,99941 \\
\hline & $\left(n I \lambda / \lambda_{0} \tau\right)_{\mathrm{mi}}$ & 0,53 & & & 14,0 & 1,30 & 0,97 & 0,38 & 1,34 & 18,8 & 21,0 & 210,0 & 10,0 & 13,0 & 1,12 & 3,35 & 0,99967 \\
\hline & $\left(n I \lambda / \lambda_{0}\right)_{\min }$ & n 0,47 & & & $\overline{17,9}$ & 1,33 & 1,18 & 0,37 & 1,18 & 21,1 & 17,8 & 208,6 & 11,7 & 15,6 & 0,845 & 2,53 & 0,99975 \\
\hline & $\lambda_{\min }$ & 0,40 & & & 29,3 & 1,62 & 1,61 & 0,31 & 1,0 & 29,3 & 19,6 & 261,1 & 13,3 & 21,5 & 0,67 & 2,0 & 0,99980 \\
\hline \multirow{5}{*}{40} & $Q_{0 \max }$ & 1,0 & \multirow{5}{*}{40,4} & \multirow{5}{*}{1,255} & 16,0 & 2,3 & 1,84 & 0,22 & 1,25 & 20,0 & 244 & 1269 & 5,2 & 12,0 & 12,2 & 36,7 & 0,9963 \\
\hline & $(n I)_{\min }$ & 0,71 & & & 20,8 & 1,46 & 1,65 & 0,34 & 0,89 & 18,7 & 74,8 & 471 & 6,3 & 9,2 & 4,0 & 12,0 & 0,9985 \\
\hline & $\left(n I \lambda / \lambda_{0} \tau\right)_{\mathrm{mi}}$ & 0,53 & & & 28,0 & 1,30 & 1,94 & 0,38 & 0,67 & 18,8 & 42 & 420 & 8,4 & 10,9 & 2,24 & 6,7 & 0,99933 \\
\hline & $\left(n I \lambda / \lambda_{0}\right)_{\min }$ & 0,47 & & & 35,9 & 1,33 & 2,26 & 0,37 & 0,59 & 21,2 & 35,8 & 376 & 9,5 & 12,6 & 1,69 & 5,1 & 0,99949 \\
\hline & $\lambda_{\min }$ & 0,40 & & & 59,6 & 1,62 & 3,10 & 0,31 & 0,53 & 31,6 & 42,3 & 474 & 11,2 & 18,1 & 1,34 & 4,0 & 0,99960 \\
\hline
\end{tabular}


With an increase in the relative operating current at a temperature drop and heat load $Q_{0}=0,5 \mathrm{~W}$ for different geometry of thermoelement legs (ratio $l / S=4,5 ; 10$; $20 ; 40)$ :

- the number of thermoelements decreases $n$ (Fig. 3). With an increase in the ratio $l / S$ , the number of thermoelements $n$ increases at a fixed relative operating current $B$; - the value of the operating current $I$ increases (Fig. 3). With an increase in the ratio $l / S$, the value of the operating current decreases $B$;

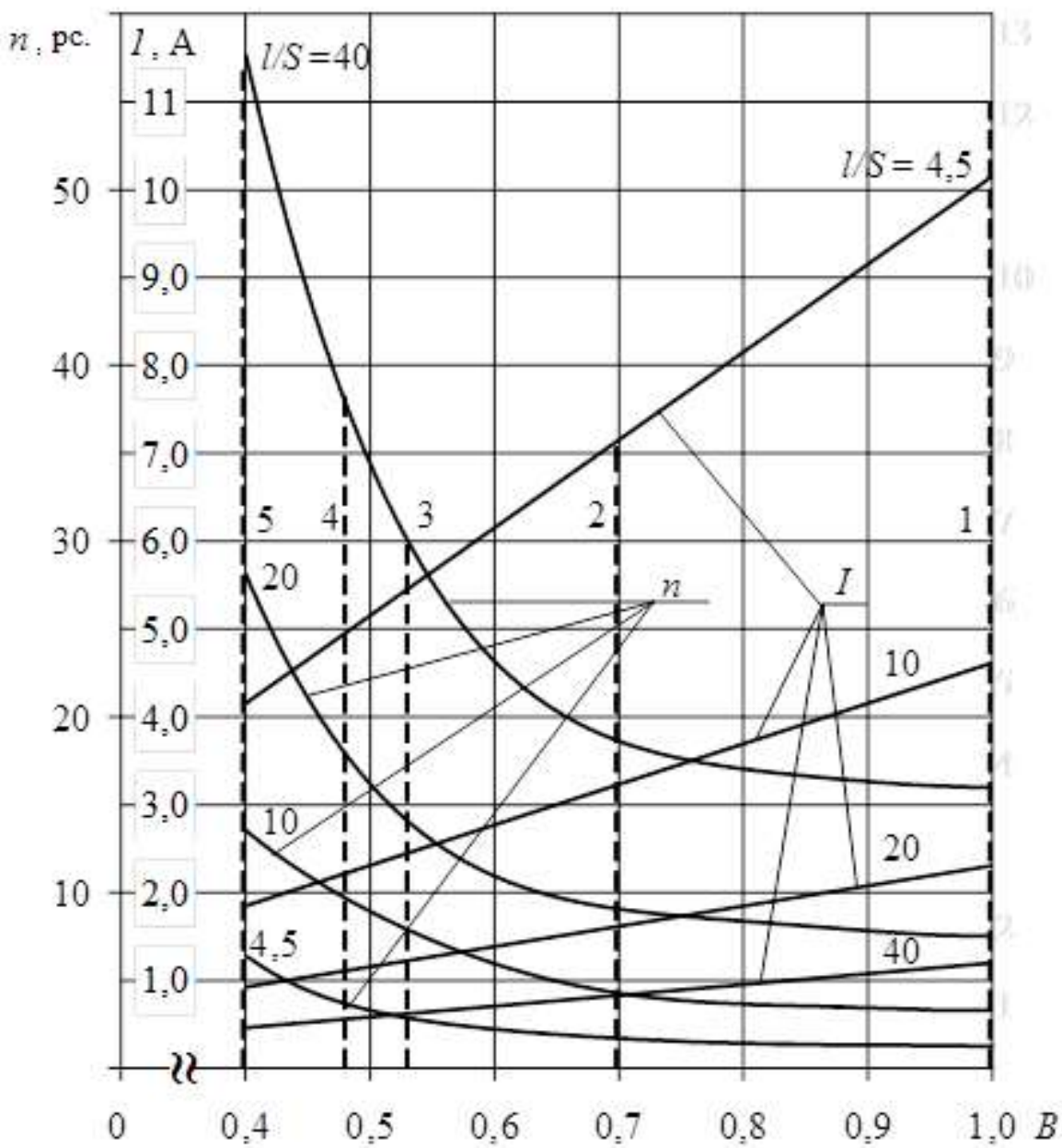

Fig. 3. Dependence of the number of thermoelements $n$ and the value $I$ of the operating current I of a single-stage TEC on the relative operating current $B$ for different geometry of the thermoelement legs $1 / \mathrm{S}$ at $T=300 \mathrm{~K}, \Delta T=40 \mathrm{~K}, Q_{0}=0,5 \mathrm{~W} .1-$ mode $Q_{0 \text { max }} ; 2-$ mode $(n I)_{\min } ; 3-\operatorname{mode}\left(n I \lambda / \lambda_{0} \tau\right)_{\min } ; 4-\operatorname{mode}\left(n I \lambda / \lambda_{0}\right)_{\min } ; 5-$ mode $\lambda_{\text {min }}$. 
- functional dependence of the complex $\left(n I \lambda / \lambda_{0} \tau\right)=f(B)$ has a minimum at $B=0,53$ (Fig. 4) for different geometry of thermoelement legs (ratio $l / S=4,5 ; 10$; $20 ; 40)$. With an increase in the ratio $l / S$, the value of the complex $\left(n I \lambda / \lambda_{0} \tau\right)$ increases at a fixed relative operating current $B$;

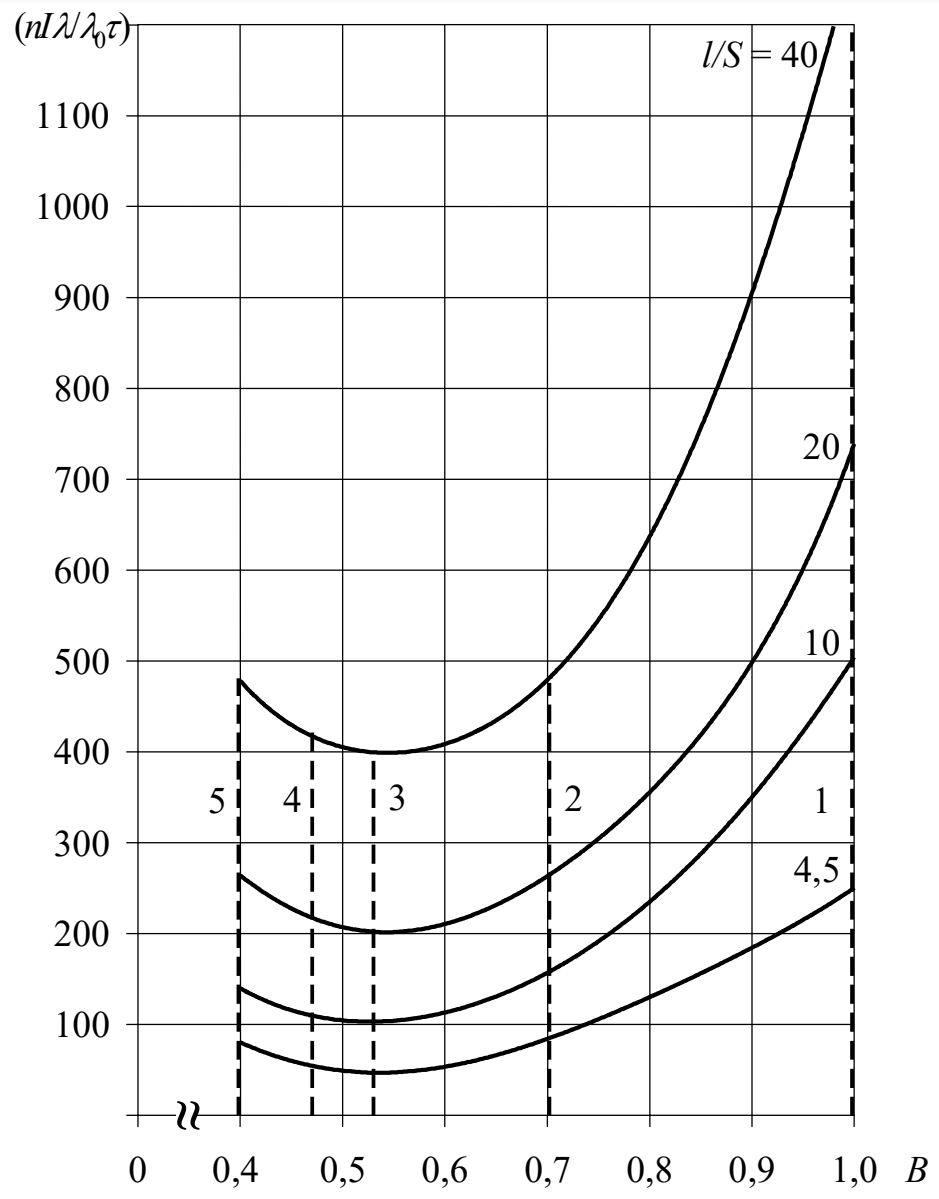

Fig. 4. Dependence of the value of a single-stage TEC complex $\left(n I \lambda / \lambda_{0} \tau\right)$ on the relative operating current $B$ for different geometry of thermoelement legs $1 / \mathrm{S}$ at $\mathrm{T}=$ $300 \mathrm{~K}, \mathrm{Q}_{0}=0,5 \mathrm{~W}, \Delta \mathrm{T}=40 \mathrm{~K} .1-\operatorname{mode} Q_{0 \text { max }} ; 2-\operatorname{mode}(n I)_{\min } ; 3-$ mode $\left(n I \lambda / \lambda_{0} \tau\right)_{\min } ; 4-\operatorname{mode}\left(n I \lambda / \lambda_{0}\right)_{\min } ; 5-\operatorname{mode} \lambda_{\min }$.

- the time of reaching the stationary operating mode $\tau$ decreases (Fig. 5) for different geometry of thermoelement legs (ratio $l / S$ ). With an increase in the ratio $l / S$, the time to reach the stationary mode of operation $\tau$ decreases at a fixed relative operating current $B$ 


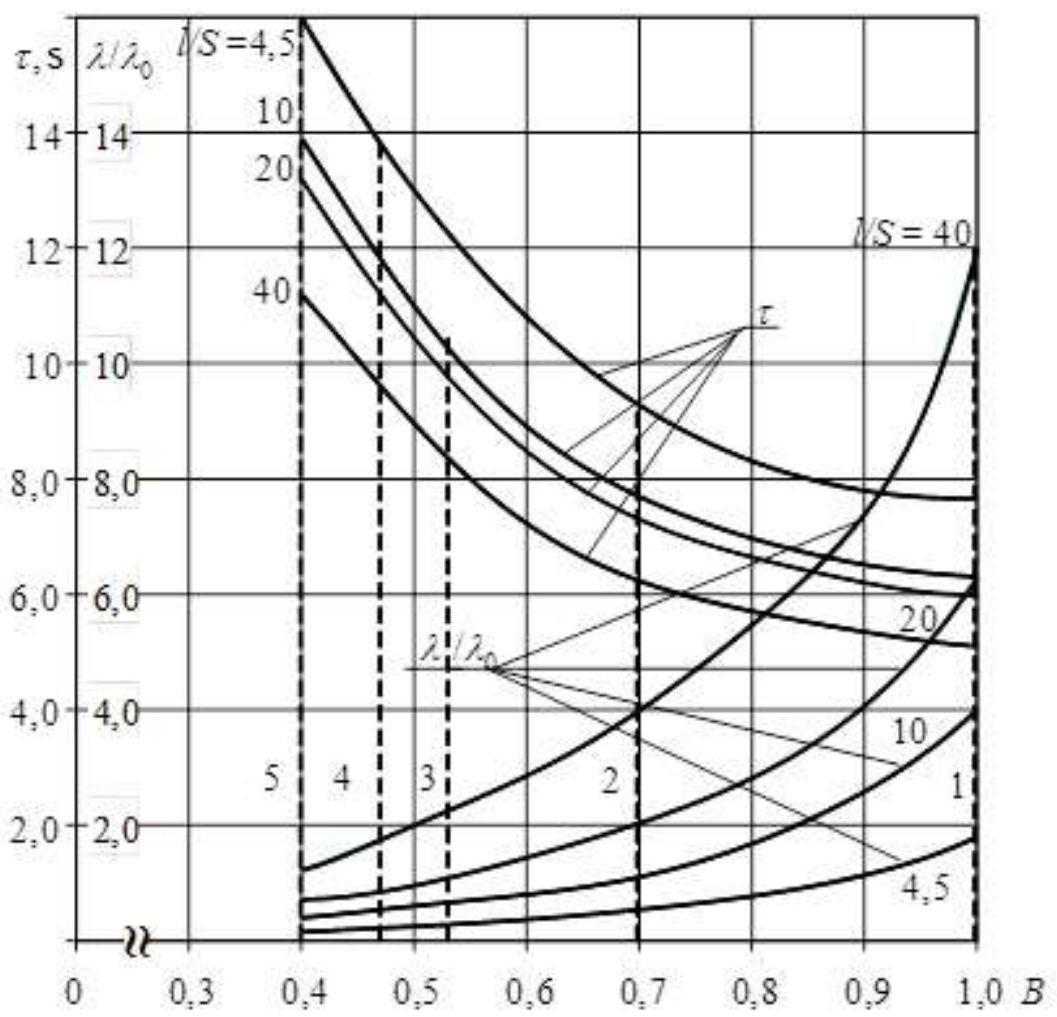

Fig. 5. Dependence of the time to reach the stationary operating mode $\tau$ and the relative value of the failure rate $\lambda / \lambda_{0}$ of a single-stage TEC on the relative operating current $B$ for different geometry of thermoelement legs $l / S$ at $T=300 K, \Delta T=$ $40 K, Q_{0}=0,5 \mathrm{~W} \quad 1$ - mode $Q_{0 \max } ; 2$ - mode $Q_{0 \max } ; 3$ - mode $\left(n I \lambda / \lambda_{0} \tau\right)_{\min } ; 4-$ mode $\left(n I \lambda / \lambda_{0}\right)_{\min } ; 5-$ mode $\lambda_{\min }$.

- the relative failure rate $\lambda / \lambda_{0}$ increases (Fig. 5) for different geometry of thermoelement legs (ratio $l / S$ ). As the ratio $l / S$ increases, the relative failure rate $\lambda / \lambda_{0}$ increases at a fixed relative operating current $B$;

- the functional dependence of the voltage drop $U=f(B)$ has a minimum at $B=0,71$ in the mode $(n I)_{\min }$ (Fig. 6) for different geometry of thermoelement legs (ratio $l / S$ ). With an increase in the ratio $l / S$, the magnitude of the voltage drop $U$ increases at a fixed relative operating current $B$; 


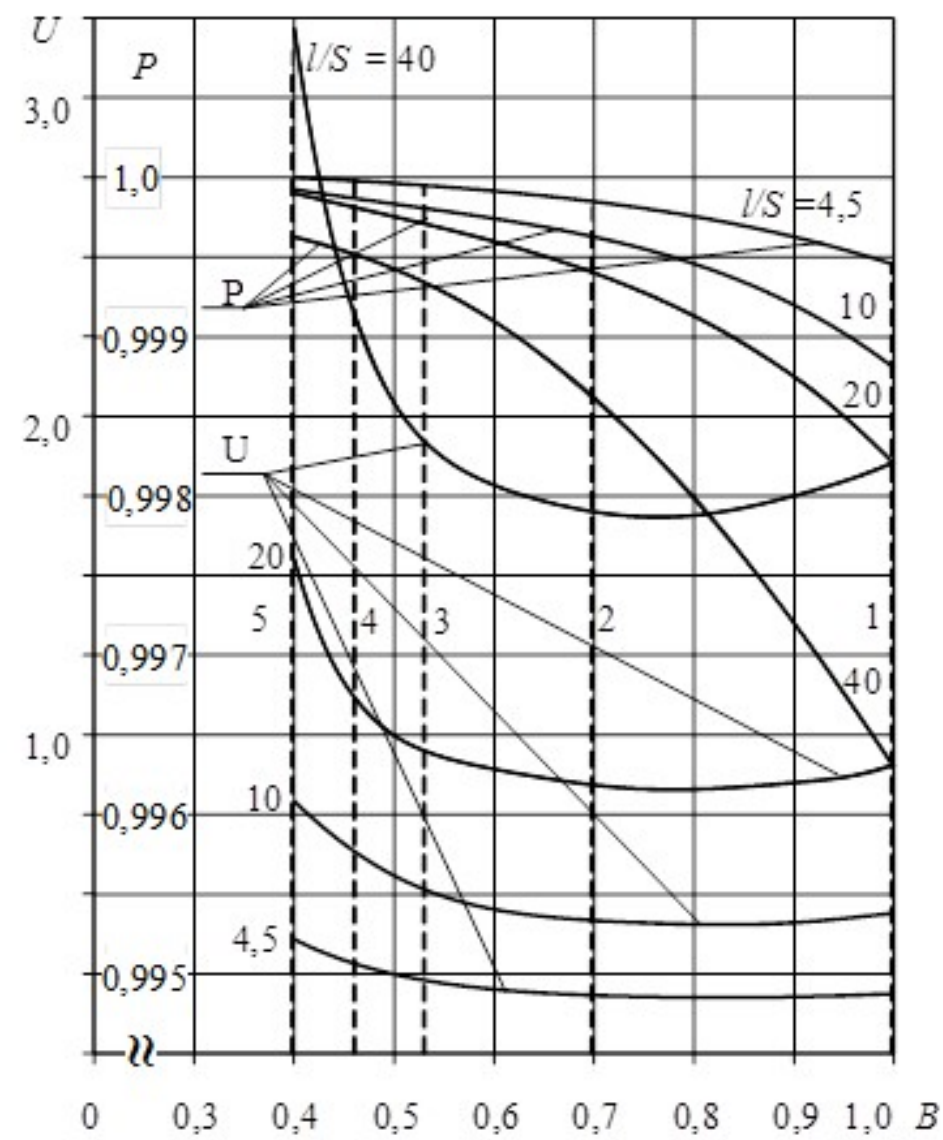

Fig. 6. Dependence of the voltage drop $U$ and the probability of failure-free operation $P$ of a single-stage TEC on the relative operating current $B$ for different geometry of thermoelement legs $l / S$ at $=300 K, \Delta T=40 K, Q_{0}=0,5 \mathrm{~W} .1-$ mode $Q_{0 \max } ; 2-$ mode $(n I)_{\min } ; 3$ - mode $\left(n I \lambda / \lambda_{0} \tau\right)_{\min } ; 4$ - mode $\left(n I \lambda / \lambda_{0}\right)_{\min } ; 5$ - mode $\lambda_{\min }$.

- the probability of failure-free operation $P$ decreases (Fig. 6) for different geometry of thermoelement legs (ratio $l / S$ ). With an increase in the ratio $l / S$, the probability of failure-free operation $P$ decreases at a fixed relative operating current $B$

- the functional dependence of the coefficient of performance $E=f(B)$ has a maximum $B=0,54$ at in the mode $\left(n I \lambda / \lambda_{0} \tau\right)_{\min }$ (Fig. 7, item 1) and does not depend on the geometry of the thermoelement legs (ratio $l / S$ ); 


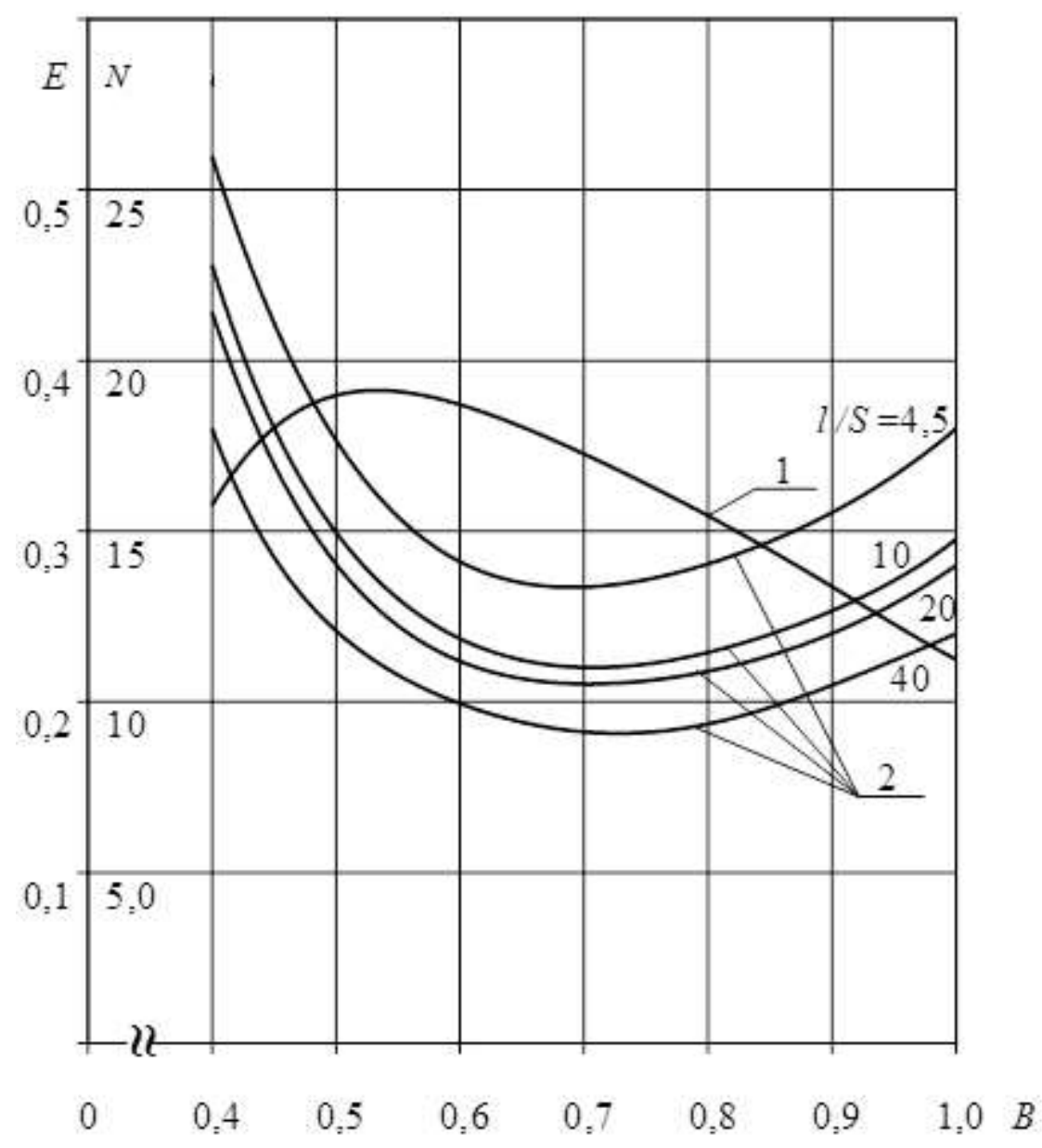

Fig. 7. Dependence of the coefficient $E$ of performance and the amount of consumed energy $N$ of a single-stage TEC on the relative operating current $B$ for different geometry of thermoelement legs (ratio $l / S$ ) at $T=300 \mathrm{~K}, \Delta T=40 \mathrm{~K}, Q_{0}=0,5 \mathrm{~W}$. $1-E=f(B), 2-N=f(B)$.

- the functional dependence of the amount of consumed energy $N=f(B)$ has a minimum $B=0,71$ at in the mode $(n I)_{\min }$ for different geometry of thermoelement legs (ratio $l / S$ ) (Fig. 7, item 2). With an increase in the ratio $l / S$, the amount of energy $N$ expended decreases at a fixed relative operating current $B$.

The calculation results of the main parameters, reliability indicators and the time to reach the stationary operating mode $\tau$ in the mode $\left(n I \lambda / \lambda_{0} \tau\right)_{\min }$ for various temperature drops $\Delta T$ from $\Delta T=10 \mathrm{~K}$ to $\Delta T=60 \mathrm{~K}$ for $l / S=4,5$ are given in Table 2. 
The main parameters and indicators in the mode $\left(n I \lambda / \lambda_{0} \tau\right)_{\min }$ at $T=300 \mathrm{~K} ; Q_{0}=0,5$ $\mathrm{W} ; \quad l / S=4,5 ; R_{H}=5 \cdot 10^{-3}$ Ohm; $I_{\operatorname{maxH}}=12,24 \mathrm{~A}$

\begin{tabular}{|c|c|c|c|c|c|c|c|c|c|c|}
\hline$B$ & $\begin{array}{c}n \\
\mathrm{pc}\end{array}$ & $\begin{array}{c}I, \\
\mathrm{~A}\end{array}$ & $\begin{array}{c}\mathrm{B} \\
\mathrm{B}\end{array}$ & $E$ & $W$, & $\begin{array}{c}\tau \\
\mathrm{s}\end{array}$ & $\begin{array}{c}N, \\
\mathrm{~W} \cdot \mathrm{s}\end{array}$ & $\lambda / \lambda_{0}$ & $\lambda \cdot 10^{8}, 1 / \mathrm{h}$ & $P$ \\
\hline$\Delta T=10 \mathrm{~K} ; T_{0}=290 \mathrm{~K} ; \Delta T_{\max }=101 \mathrm{~K} ; \Theta=0,1 ; \mathrm{R}=4,89 \cdot 10^{-3} \mathrm{Ohm} I_{\max }=12,0 \mathrm{~A}$ \\
\hline 0,113 & 6,3 & 1,36 & 0,11 & 3,37 & 0,15 & 7,9 & 1,19 & 0,00035 & 0,0011 & 0,99999989 \\
\hline \multicolumn{8}{|c|}{$\Delta T=20 \mathrm{~K} ; T_{0}=280 \mathrm{~K} ; \Delta T_{\max }=93,7 \mathrm{~K} ; \Theta=0,213 ; \mathrm{R}=4,74 \cdot 10^{-3} 0 \mathrm{hm}$} \\
\hline 0,237 & 3,7 & 2,80 & 0,13 & 1,41 & 0,355 & 8,9 & 3,16 & 0,0072 & 0,0216 & 0,9999978 \\
\hline \multicolumn{8}{|c|}{$\Delta T=30 \mathrm{~K} ; T_{0}=270 \mathrm{~K} ; \Delta T_{\max }=86,8 \mathrm{~K} ; \Theta=0,346 ; \mathrm{R}=4,69 \cdot 10^{-3} \mathrm{Ohm}$} \\
\hline 0,376 & 3,1 & 4,31 & 0,16 & 0,71 & 0,70 & $10,0,5$ & 7,0 & 0,052 & 0,156 & 0,999984 \\
\hline$\Delta T=40 \mathrm{~K} ; T_{0}=260 \mathrm{~K} ; \Delta T_{\max }=79,8 \mathrm{~K} ; \Theta=0,50 ; \mathrm{R}=4,55 \cdot 10^{-3} \mathrm{Ohm}$ & $I_{\max }=11,1 \mathrm{~A}$ \\
\hline 0,532 & 3,2 & 5,9 & 0,22 & 0,38 & 1,30 & 11,9 & 15,5 & 0,255 & 0,766 & 0,999923 \\
\hline \multicolumn{8}{|c|}{$\Delta T=50 \mathrm{~K} ; T_{0}=250 \mathrm{~K} ; \Delta T_{\max }=73,1 \mathrm{~K} ; \Theta=0,684 ; \mathrm{R}=4,41 \cdot 10^{-3} 0 \mathrm{hm}$} \\
\hline 0,71 & 4,1 & 7,7 & 0,36 & 0,18 & 2,77 & 15,0 & 41,6 & 1,12 & 3,36 & 0,99966 \\
\hline$\Delta T=60 \mathrm{~K} ; T_{0}=240 \mathrm{~K} ; \Delta T_{\max }=66,8 \mathrm{~K} ; \Theta=0,90 ; \mathrm{R}=4,33 \cdot 10^{-3} \mathrm{Ohm}$ & $I_{\max }=10,53 \mathrm{~A}$ \\
\hline 0,91 & 11,3 & 9,6 & 1,2 & 0,044 & 11,5 & 23,1 & 266 & 8,3 & 24,8 & 0,9975 \\
\hline
\end{tabular}

With an increase in the temperature difference $\Delta T$ in the mode $\left(n I \lambda / \lambda_{0} \tau\right)_{\min }$ at $=0.5 \mathrm{~W}$ and the ratio $l / S=4,5$ :

- the relative operating current $B$ increases (Fig. 8, item 1);

- functional dependence $n=f(\Delta T)$ has a minimum at $\Delta T=30 \mathrm{~K}$ (Fig. 8 item 2);

- the refrigerating coefficient $E$ decreases (Fig. 8, item 3);

- the time of reaching the stationary mode of operation $\tau$ increases (Fig. 9, item 1);

- the relative intensity of failures $\lambda / \lambda_{0}$ increases (Fig. 9, item 2);

- the probability of failure-free operation $P$ decreases (Fig. 9, item 3);

- the amount of energy expended increases $N$. 


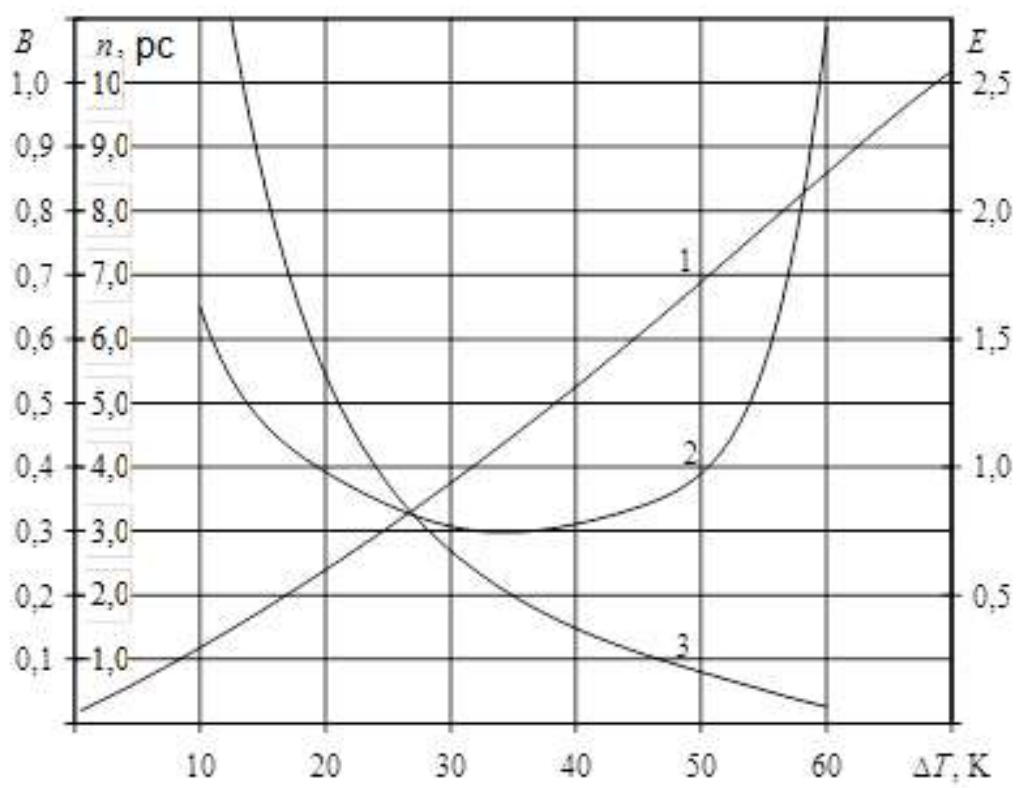

Fig. 8. Dependence of the coefficient $E$ of performance and the amount of consumed energy $N$ of a single-stage TEC on the relative operating current $B$ for different geometry of thermoelement legs (ratio $l / S$ ) at $T=300 \mathrm{~K}, \Delta T=40 \mathrm{~K}, Q_{0}=0,5 \mathrm{~W}$. $1-E=f(B), 2-N=f(B)$.

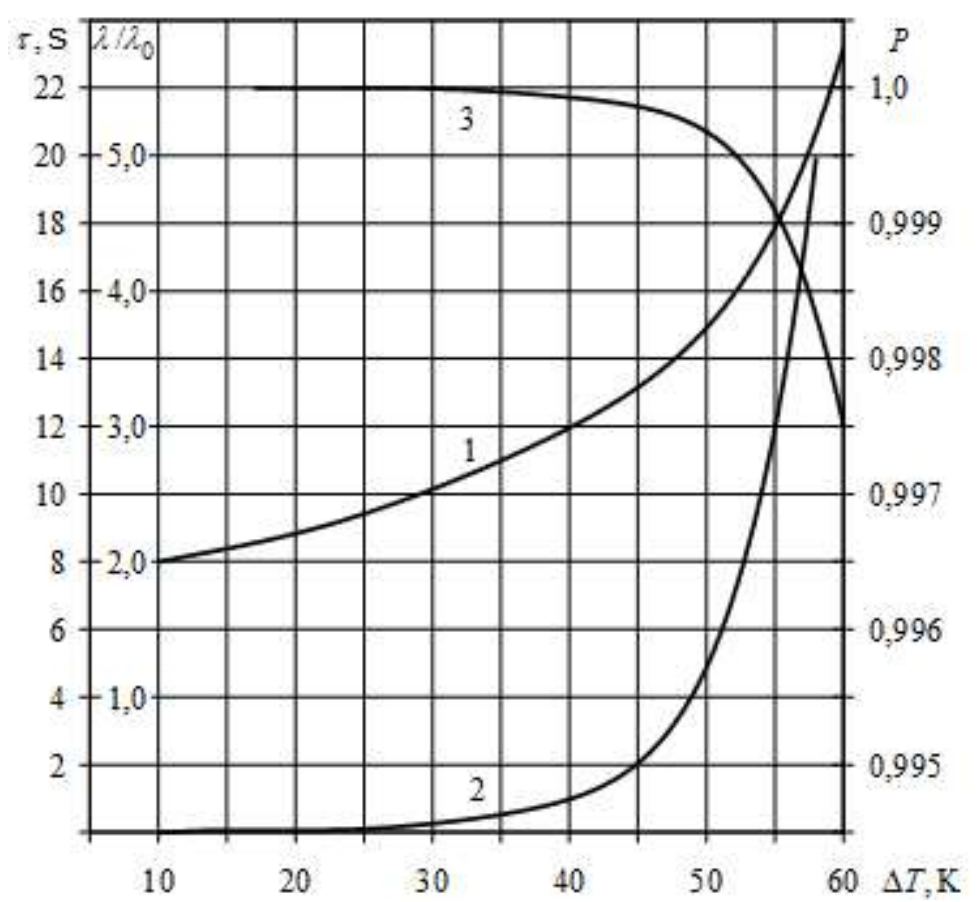

Fig. 9. Dependence of the time of reaching the stationary mode of operation $\tau$, the relative magnitude of the failure rate $\lambda / \lambda_{0}$, the probability of failure-free operation $P$ of a single-stage TEC on the temperature difference $\Delta T$ in the mode $\left(n I \lambda / \lambda_{0} \tau\right)_{\min }$ at $T=300 \mathrm{~K}, Q_{0}=0,5 \mathrm{~W}, l / s=4,5 \quad 1-\tau=f(\Delta T), 2-\lambda / \lambda_{0}=f(\Delta T), 3-$ $P=f(\Delta T)$ 
Conclusions:

1. A model for controlling the thermal regime of a single-stage TEC with minimizing the complex $\left(n I \lambda / \lambda_{0} \tau\right)$ in the range of temperature drops from $\Delta T=10 \mathrm{~K}$ to $\Delta T=60 \mathrm{~K}$ and stationary heat load $Q_{0}=0,5 \mathrm{~W}$.

2. Received a relationship to determine the optimal relative operating current $B_{\text {опт }}$ corresponding to the minimum value of the complex $\left(n I \lambda / \lambda_{0} \tau\right)_{\min }$.

3. At a given temperature drop and stationary heat load $Q_{0}$ the size of the complex $\left(n I \lambda / \lambda_{0} \tau\right)$ depends on the geometry of the thermoelement legs $l / S$.

4. The minimization of the complex $\left(n I \lambda / \lambda_{0} \tau\right)$ with the variation of the four main parameters $n, I, \lambda / \lambda_{0}$ and $\tau$ allows you to select the current mode of operation that provides optimal control of the thermal mode of the TPP with the minimum power consumption with acceptable weight and size, reliability and dynamic characteristics of the device. 